J. Environ. Sci.

Institute of Environmental Studies and Research - Ain Shams University

\title{
COMPARING TWO DIFFERENT ENVIRONMENTAL SOLUTIONS FOR INDUSTRIAL WASTEWATER MANAGEMENT IN QUESNA INDUSTRIAL ZONE
}

\author{
Maysara F. Ahmed ${ }^{(1)}$; Mohamed E. A. El Nadi ${ }^{(2)}$ \\ and Nahla M. Bawdy ${ }^{(2)}$
}

1) Institute of environmental studies and research, Ain Shams University 2)Faculty of Engineering, Ain Shams University

\begin{abstract}
The industrial wastewater management in Egypt is one of the main goals for sustainable development. The water crisis in Egypt has lead the industrial sector to search for resource efficiency and cleaner production technologies through a proper management of water, energy, and raw materials. As a result, the aim of this study is to compare between 2 different environmental solutions for industrial wastewater management in Quesna industrial zone in Monofeya governorate. Industrial wastewater samples were taken from ten factories representing the ten main industrial sectors in the study area and five parameters were measured in each sample.

Results achieved from the two different applied scenarios showed variation in flow rates, industrial wastewater loads, treatment methods, removal efficiencies, capital and operation costs of the applied scenarios. Finally, these scenarios were compared technically and financially to determine the best environmental solution for industrial wastewater management in Quesna industrial zone. This study could be applied for all industrial zone in Egypt using the same methodologies.

Key words: Industrial Wastewater Management (IWWM), Quesna Industrial Zone, Industrial Sectors in Quesna, Industrial Wastewater Treatment (IWWT), Environmental Solutions, Removal Efficiency, and Industrial Wastewater Load.
\end{abstract}




\section{INTRODUCTION}

After the First World War, industrial activities based on natural resources began to increase. Industries make up about $80 \%$ of the entire pollution load in wastewater, industrial and domestic activities result in the production of vast quantities of wastewater. The liquid industrial waste discharged into urban sanitation has increased both in volume and complexity during recent decades. In addition, municipal wastewater has changed in composition, not only through the increased amount of household chemicals in use but also through the discharge of varying amounts of industrial waste into public sewers.

Internationally, different studies were carried out such as the thesis presented by Toll (2009) on the current situation in Chile with regard to the treatment of industrial wastewater. This study aimed to identify the potential for the implementation of separation technologies. Another important study was carried out by Saikku (2006) who mentioned that the concept of an ecoindustrial park is a sector of industrial ecology, which draws analogies from natural ecosystems to human industrial systems as Kalundborg in Denmark. Her study serves as a background for the first planned eco-industrial park in Finland, at Rantasalmi municipality which involves mainly small mechanical wood processing companies.

In Italy, Tessitore et al. (2015) studied the evolution of eco- industrial parks and stated that it is a development opportunity for many territories and companies where the key element of Italian eco-industrial parks is the management body, an entity provided by national legislation to manage and 
coordinate companies and to develop more environmentally sustainable production practices. The study introduced an important environmental management experience implemented in Italy.

In China, Liu and Côté (2017) carried out another study on the case of eco-industrial parks, where they considered them critical for sustainable development, ecosystem services are increasingly being put on the policy agendas of governments and corporations in China.

Rio de Janeiro showed an important study carried out by Veiga et al. (2008) who stated that the eco-industrial park is an environmental management tool that is being spread in many nations as an industrial model that can reconcile the three "Es" of sustainability - environment, social equity and economic efficiency - as it reorganizes industrial practices and activities in order to meet sustainable development goals. In addition, it is a tool towards the reduction, reuse and recycling (3R's) of by-products and wastes.

In Egypt, many previous studies discussed the treatment types of Industrial Wastewater (IWW) of different sectors. Some of which are: Mohamed (2012), who studied treatment of painting IWW, Ashor (2013), who focused in her study on treatment of liquid wastes produced by ceramic industry, and El-Hefny (2018), who made an important study on upgrading of existing dairy IWWTP. These studies discussed the treatment of IWW in different factories but did not include management of IWW for a specific industrial sector or for an industrial zone.

On the other hand, the current study is the first one to discuss the management system of IWW in one of the Egyptian industrial zones, where Mubarak Industrial Zone in Quesna City has ten main industrial sectors, they 
are: Plastic sector including 19 companies, Pulp and Paper sector including 3 large companies and 10 small companies, Chemicals sector including 11 companies, Electric and Engineering sector including 13 companies, Agricultural sector including 14 companies, Textile sector including 28 companies, Metals sector including 10 companies, Food sector including 3 large companies and 27 small companies, Tanneries sector including 3 large companies and 4 small companies, and Pharmaceutical sector including 5 companies (Investment Map of Monofeya Governorate, 2012).

Because of increasing demand and pressure on natural resources by growing human population, the main objective of this study is comparing between the effective solution of industrial wastewater management and the existing industrial wastewater management in Quesna industrial zone to achieve the sustainable development and water conservation.

\section{MATERIALS AND METHODS}

This study focused on Quesna industrial zone located in Monofeya governorate. This industrial zone is representative for Egyptian industrial zones including ten main industrial sectors representing 150 factories (Investment Map of Monofeya Governorate, 2012) where two different scenarios for industrial wastewater management are proposed in this study to choose the best environmental solution. Ten samples were taken from ten different factories representing the ten main industrial sectors in Quesna industrial zone. These sectors are: food, pharmaceutical, chemicals, electric \& engineering, metals, plastic, pulp \& paper, agriculture, and textile sectors. 
A sample was taken twice a week with a total of eight samples per month. Different parameters were measured during one year 2016 including BOD, COD, heavy metals (Ni, Cr, and Zn), TSS and TDS. Each parameter was measured with certain equipment according to the American Standard Methods for the analysis of water and wastewater (2009). The industrial wastewater flow rate for each industrial sector was measured per day then the average value was calculated per each month in $\mathrm{m} 3 /$ day. The industrial wastewater load for existing situation in each sector is calculated by multiplying the maximum value for each parameter by the flow rate average value in each month per day. The study proposed two scenarios to manage the industrial wastewater generated from Quesna industrial zone. These scenarios are:

Scenario (1): proposes that all companies will discharge their IWW without any treatment to one centralized IWWTP.

Scenario (2): proposes that the industrial sectors having similar industrial wastewater characteristics were grouped together to be treated before being discharged to the centralized IWWTP.

The outlet of the IWWTP for the two scenarios will be used for agricultural usage. The parameters for the outlet of the IWWTP will be presented in the table (1). 
Table (1): Specifications for Agricultural usage (Law 48/ 1982)

\begin{tabular}{|c|c|}
\hline Parameters & Values \\
\hline \hline TDS & 1,000 \\
\hline TSS & 30 \\
\hline BOD & 30 \\
\hline COD & 50 \\
\hline $\mathrm{Ni}$ & 0.1 \\
\hline $\mathrm{Cr}$ & 0.05 \\
\hline $\mathrm{Zn}$ & 2 \\
\hline
\end{tabular}

RESULTS AND DISCUSSION

Surveying Quesna industrial zone, resulted in finding one centralized industrial wastewater treatment plant. The maximum value of flow entering the existing centralized wastewater treatment plant in Quesna industrial zone equals 30,000 m3/ day. This flow includes the domestic wastewater for three villages (Al-Manashy, Kofour Al-Raml, and Al-Khawagah) with amount equals $6,000 \mathrm{~m} 3 /$ day. The expected results for applying the two proposed scenarios to manage the industrial wastewater generated from Quesna industrial zones as follow:

SCENARIO 1: In this scenario, we propose that all companies will discharge their IWW without any treatment to one centralized IWWTP as showing in the figure (1) below. The load of discharging into the centralized IWWTP and the maximum flow rate in $\mathrm{m} 3$ / day is presented in table (2), while the table (3) is showing the back calculation to the concentration in $\mathrm{mg} / \mathrm{l}$ which will enter the centralized IWWTP and the expected outputs after applying the required treatment. 
The centralized IWWTP will include two stages phyisco-chemical treatment unit and biological treatment unit to achieve the permissible limits for agriculture usage. In this scenario, no sewage network is needed and transportation is not needed as well. Regarding companies that store the untreated wastewater in large tanks should have their own sewage network in order to discharge their IWW to the main network. The removal efficiency of the centralized IWWP is presented in table (3).

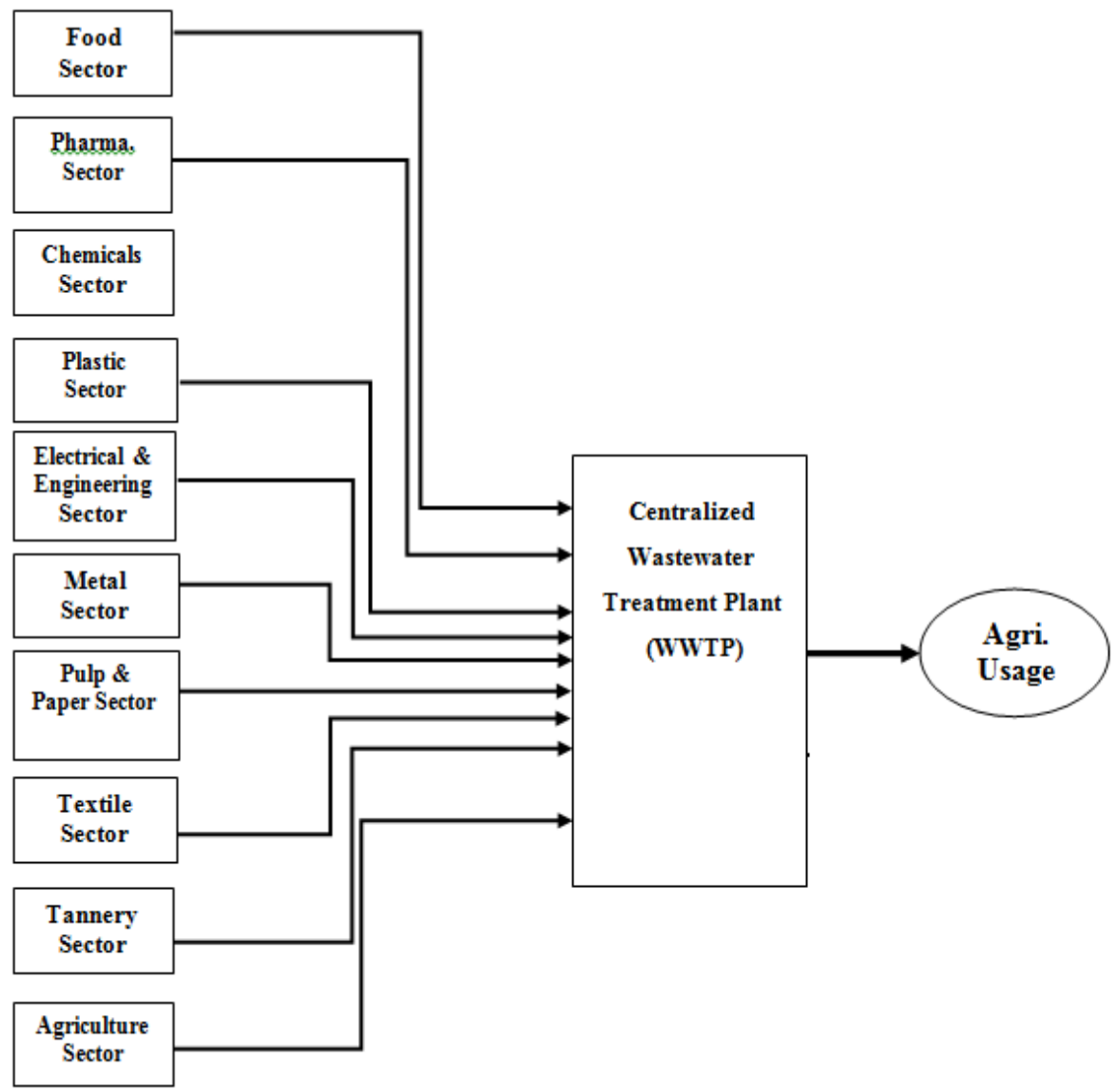

Figure (1): Scheme for Scenario (1) 
Table (2): The estimated industrial wastewater load and max. flow rate in Scenario 1

\begin{tabular}{|c|c|c|c|c|c|c|c|c|c|c|}
\hline & & 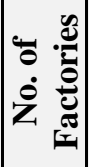 & 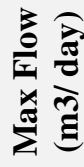 & 苗 & की & 光 & 己ٍ & 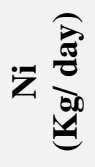 & $=\frac{\hat{\vec{\theta}}}{\hat{\theta}}$ & 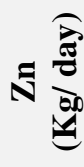 \\
\hline \multirow{3}{*}{$\begin{array}{l}\text { Large Food } \\
\text { Sector }\end{array}$} & Min & \multirow{3}{*}{3} & \multirow{3}{*}{900} & 504 & 720 & 360 & 675 & 0 & 0 & 0 \\
\hline & Max & & & 1944 & 1845 & 1467 & 3103.20 & 0 & 0 & 0 \\
\hline & Ave & & & 1181.93 & 1287.38 & 909.48 & 1875.52 & 0 & 0 & 0 \\
\hline \multirow{3}{*}{$\begin{array}{l}\text { Small Food } \\
\text { Sector }\end{array}$} & Min & \multirow{3}{*}{27} & \multirow{3}{*}{945} & 573.48 & 107.33 & 582.39 & 1358.78 & 0 & 0 & 0 \\
\hline & $\operatorname{Max}$ & & & 1334.34 & 344.25 & 1168.83 & 3178.04 & 0 & 0 & 0 \\
\hline & Ave & & & 880.57 & 815.54 & 847.17 & 2094.61 & 0 & 0 & 0 \\
\hline \multirow{3}{*}{$\begin{array}{c}\text { Pharma. } \\
\text { Sector }\end{array}$} & Min & \multirow{3}{*}{5} & \multirow{3}{*}{1800} & 834.40 & 1168 & 1452 & 4192 & 0 & 0 & 0 \\
\hline & Max & & & 1222.77 & 12274.50 & 7992 & 16143.75 & 0 & 0 & 0 \\
\hline & Ave & & & 1088.53 & 7684.23 & 4992.24 & 10231.81 & 0 & 0 & 0 \\
\hline \multirow{3}{*}{$\begin{array}{c}\text { Chemicals } \\
\text { Sector }\end{array}$} & Min & \multirow{3}{*}{11} & \multirow{3}{*}{3850} & 4090.24 & 4147.61 & 1669.36 & 2688.84 & 0 & 0 & 0 \\
\hline & Max & & & 5120.50 & 5882.80 & 2079 & 3349.50 & 0 & 0 & 0 \\
\hline & Ave & & & 4473.52 & 5043.53 & 1814.83 & 2924.30 & 0 & 0 & 0 \\
\hline
\end{tabular}

Followed Table (2): The estimated industrial wastewater load and max. flow rate in Scenario 1

\begin{tabular}{|c|c|c|c|c|c|c|c|c|c|c|}
\hline & & 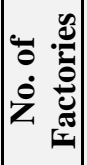 & 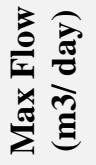 & 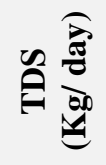 & 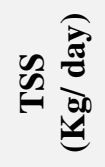 & மำ & 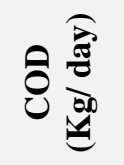 & 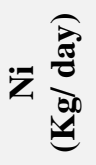 & v & 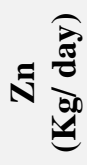 \\
\hline \multirow{3}{*}{$\begin{array}{l}\text { Plastic } \\
\text { Sector }\end{array}$} & Min & \multirow{3}{*}{19} & \multirow{3}{*}{760} & 3170.91 & 5856.18 & 4950.45 & 88196.60 & 0 & 0 & 0 \\
\hline & Max & & & 3531.72 & 7815.08 & 6606.68 & 10940.20 & 0 & 0 & 0 \\
\hline & Ave & & & 3366.10 & 6677.69 & 5636.94 & 9343.81 & 0 & 0 & 0 \\
\hline \multirow{3}{*}{$\begin{array}{c}\text { Electric \& } \\
\text { Engineering } \\
\text { Sector }\end{array}$} & Min & \multirow{3}{*}{13} & \multirow{3}{*}{650} & 318.73 & 188.37 & 166.73 & 258.51 & 3.73 & 0.01 & 3.33 \\
\hline & Max & & & 624 & 331.50 & 211.25 & 344.50 & 5.07 & 0.04 & 4.27 \\
\hline & Ave & & & 378.80 & 274.27 & 187.59 & 313.63 & 4.52 & 0.02 & 3.95 \\
\hline \multirow{3}{*}{$\begin{array}{l}\text { Large } \\
\text { Tannery } \\
\text { Sector }\end{array}$} & Min & \multirow{3}{*}{3} & \multirow{3}{*}{5,100} & 5625 & 8739 & 4437 & 10638 & 0 & 2245.50 & 0 \\
\hline & Max & & & 6415.80 & 9934.80 & 5049 & 12066.60 & 0 & 2524.50 & 0 \\
\hline & Ave & & & 6209.07 & 9604.01 & 4880.47 & 11698.05 & 0 & 2439.44 & 0 \\
\hline \multirow{3}{*}{$\begin{array}{l}\text { Small } \\
\text { Tannery } \\
\text { Sector }\end{array}$} & Min & \multirow{3}{*}{4} & \multirow{3}{*}{1,200} & 1250 & 1814.80 & 982 & 2360 & 0 & 499 & 0 \\
\hline & Max & & & 1509.60 & 2220 & 1182 & 2839.20 & 0 & 595.20 & 0 \\
\hline & Ave & & & 1392 & 2026.05 & 1091.01 & 2622.52 & 0 & 0 & 0 \\
\hline \multirow{3}{*}{$\begin{array}{l}\text { Metals } \\
\text { Sector }\end{array}$} & Min & \multirow{3}{*}{10} & \multirow{3}{*}{600} & 3947.40 & 2135.16 & 969.30 & 1453.14 & 0 & 0 & 0.27 \\
\hline & Max & & & 4398 & 2371.20 & 1081.20 & 1620 & 0.06 & 302.40 & 0.42 \\
\hline & Ave & & & 4165.23 & 2251.88 & 1023.23 & 1533.46 & 0.01 & 280.56 & 0.35 \\
\hline
\end{tabular}


J. Environ. Sci.

Institute of Environmental Studies and Research - Ain Shams University

Followed Table (2): The estimated industrial wastewater load and max. flow rate in Scenario 1

\begin{tabular}{|c|c|c|c|c|c|c|c|c|c|c|}
\hline & & 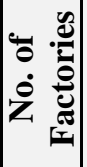 & 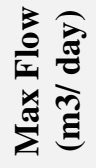 & 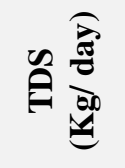 & 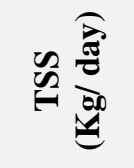 & คै & 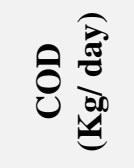 & $=\frac{\widehat{\vec{\sigma}}}{\underline{\partial}}$ & $=\frac{\widehat{\vec{\sigma}}}{\sqrt{0}}$ & 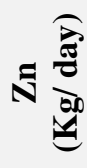 \\
\hline \multirow{3}{*}{$\begin{array}{c}\text { Large Pulp } \\
\text { \& paper } \\
\text { Sector }\end{array}$} & Min & \multirow{3}{*}{3} & \multirow{3}{*}{4,500} & 31863.75 & 47989.20 & 21845.70 & 32416.20 & 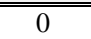 & 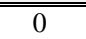 & 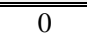 \\
\hline & Max & & & 38594.25 & 63702 & 22648.50 & 33592.50 & 0 & 0 & 0 \\
\hline & Ave & & & 36685.20 & 44794.71 & 22402.77 & 33247.83 & 0 & 0 & 0 \\
\hline \multirow{3}{*}{$\begin{array}{c}\text { Small Pulp } \\
\text { \& paper } \\
\text { Sector }\end{array}$} & Min & \multirow{3}{*}{10} & \multirow{3}{*}{350} & 2086.26 & 3514.70 & 1608.96 & 2382.40 & 0 & 0 & 0 \\
\hline & Max & & & 3265.15 & 3977.82 & 1761.55 & 2612.75 & 0 & 0 & 0 \\
\hline & Ave & & & 2625.50 & 3734.14 & 1712.48 & 2541.54 & 0 & 0 & 0 \\
\hline \multirow{3}{*}{$\begin{array}{l}\text { Agriculture } \\
\text { Sector }\end{array}$} & Min & \multirow{3}{*}{14} & \multirow{3}{*}{2,520} & 1734.60 & 1734.60 & 5744.76 & 8506.40 & 0 & 0 & 0 \\
\hline & Max & & & 2472.12 & 2227.68 & 7396.20 & 10962 & 0 & 0 & 0 \\
\hline & Ave & & & 2096.62 & 1988.37 & 6611.82 & 9805.19 & 0 & 0 & 0 \\
\hline \multirow{3}{*}{$\begin{array}{l}\text { Textile } \\
\text { Sector }\end{array}$} & Min & \multirow{3}{*}{28} & \multirow{3}{*}{840} & 2205 & 6720 & 1170.40 & 2394 & 0 & 0 & 0.21 \\
\hline & Max & & & 2657.76 & 17220 & 1404.48 & 2874.48 & 0.01 & 0.01 & 0.26 \\
\hline & Ave & & & 2473.70 & 11442.64 & 1304.45 & 2634.24 & 0 & 0.01 & 0.23 \\
\hline \multirow{3}{*}{ Total } & Min & \multirow{3}{*}{150} & \multirow{3}{*}{24,015} & 58203.77 & 84834.94 & 45939.05 & 77519.86 & 3.74 & 3007 & 3.81 \\
\hline & Max & & & 73090.01 & 130146.63 & 60047.69 & 103626.72 & 5.14 & 3422 & 4.95 \\
\hline & Ave & & & 65646.89 & 107490.79 & 52993.37 & 90573.29 & 4.44 & 3215 & 4.38 \\
\hline
\end{tabular}


Table (3): The estimated IWW parameters concentration which will enter the centralized IWWTP and its outputs

\begin{tabular}{|c|c|c|c|c|c|c|c|}
\hline & TDS & TSS & BOD5 & COD & $\mathbf{N i}$ & $\mathrm{Cr}$ & $\mathbf{Z n}$ \\
\hline $\begin{array}{l}\text { Concentration } \\
\text { (inlet) }(\mathrm{mg} / \mathrm{l}) \\
\end{array}$ & 3043.51 & 5419.39 & 2500.42 & 4315.08 & 0.21 & 142.50 & 0.21 \\
\hline $\begin{array}{c}\text { Pre-Treatment } \\
\text { Unit } \\
(\mathrm{mg} / \mathrm{l}) \\
\end{array}$ & 3043.51 & 3793.57 & 1875.32 & 3236.31 & 0.21 & 142.50 & 0.21 \\
\hline $\begin{array}{l}\text { Phyisco- } \\
\text { Chemical } \\
\text { Treatment Unit } \\
(1)(\mathrm{mg} / \mathrm{l})\end{array}$ & 1826.11 & 948.39 & 1031.43 & 1779.97 & 0.21 & 7.13 & 0.21 \\
\hline $\begin{array}{c}\text { Biological } \\
\text { Treatment } \\
\text { Unit }(1)(\mathrm{mg} / \mathrm{l}) \\
\end{array}$ & 1004.36 & 47.42 & 51.57 & 89 & 0.01 & 0.36 & 0.01 \\
\hline $\begin{array}{c}\text { Phyisco- } \\
\text { Chemical } \\
\text { Treatment Unit } \\
(2)(\mathrm{mg} / \mathrm{l})\end{array}$ & 602.62 & 11.85 & 28.36 & 48.95 & 0.01 & 0.02 & 0.01 \\
\hline $\begin{array}{c}\text { Biological } \\
\text { Treatment } \\
\text { Unit }(2)(\mathrm{mg} / \mathrm{l}) \\
\end{array}$ & 331.44 & 0.59 & 1.42 & 2.45 & 0 & 0 & 0 \\
\hline $\begin{array}{l}\text { Concentration } \\
\text { Outlet)( } \\
\text { (mg/ l) of Drain } \\
\text { Law } 48 / 82 \text { for } \\
\text { agriculture } \\
\text { usage }\end{array}$ & 1000 & 30 & 30 & 50 & 0.10 & 0.05 & 2 \\
\hline $\begin{array}{c}\text { Removal Eff. } \\
(\% \eta) \text { Final }\end{array}$ & 89.11 & 99.99 & 99.94 & 99.94 & 99.75 & 100 & 99.75 \\
\hline
\end{tabular}

SCENARIO 2: In this scenario, it was proposed that all companies having the same wastewater parameters in their IWW will discharge to individual treatment unit after mixing together before discharging to one Centralized IWWP to treat the final stream as showing in the figure (2) below. As a result, three individual IWWT units will be found before being discharged to the centralized IWWTP. The first unit will gather the discharge IWW from food 
and agriculture sectors, whereas the second unit will gather the discharge IWW from textile, tannery, plastic, pharmaceutical, pulp and paper sectors. Metals, chemicals, electrical and engineering sectors will discharge their IWW to the third unit. The outlet of these three individual IWWT units will be discharged together to one centralized IWWP.

The loads and max flow rate discharged to the three individual IWWT units is presented in table (4). While table (5) represents the back calculation to the concentration in $\mathrm{mg} / \mathrm{l}$ entering the individual IWWT units and the expected outputs after applying the required treatment for each unit. Table (6) will present the back calculation to the concentration in $\mathrm{mg} / 1$ entering the centralized IWWTP and the expected outputs after applying the required treatment.

The first individual treatment unit will include one stage phyiscochemical treatment unit and biological treatment unit. The second individual treatment unit will include one stage phyisco-chemical treatment unit and one biological treatment unit followed by tertiary treatment that contains filter sand unit, whereas the third individual treatment unit will include one stage phyisco-chemical treatment unit and biological treatment unit. In this scenario, transportation is needed to collect the companies of the same sector together as mentioned before. The output of the three individual treatment units will discharge to the centralized IWWTP which includes one biological treatment unit to achieve the permissible limits for agriculture usage. In addition, sewage network is needed to discharge to the three individual treatment units and then to be collected together before entering to the centralized IWWTP. The removal efficiencies of the three individual 
treatment units and the centralized IWWP are presented in tables (5) \& (6) respectively.

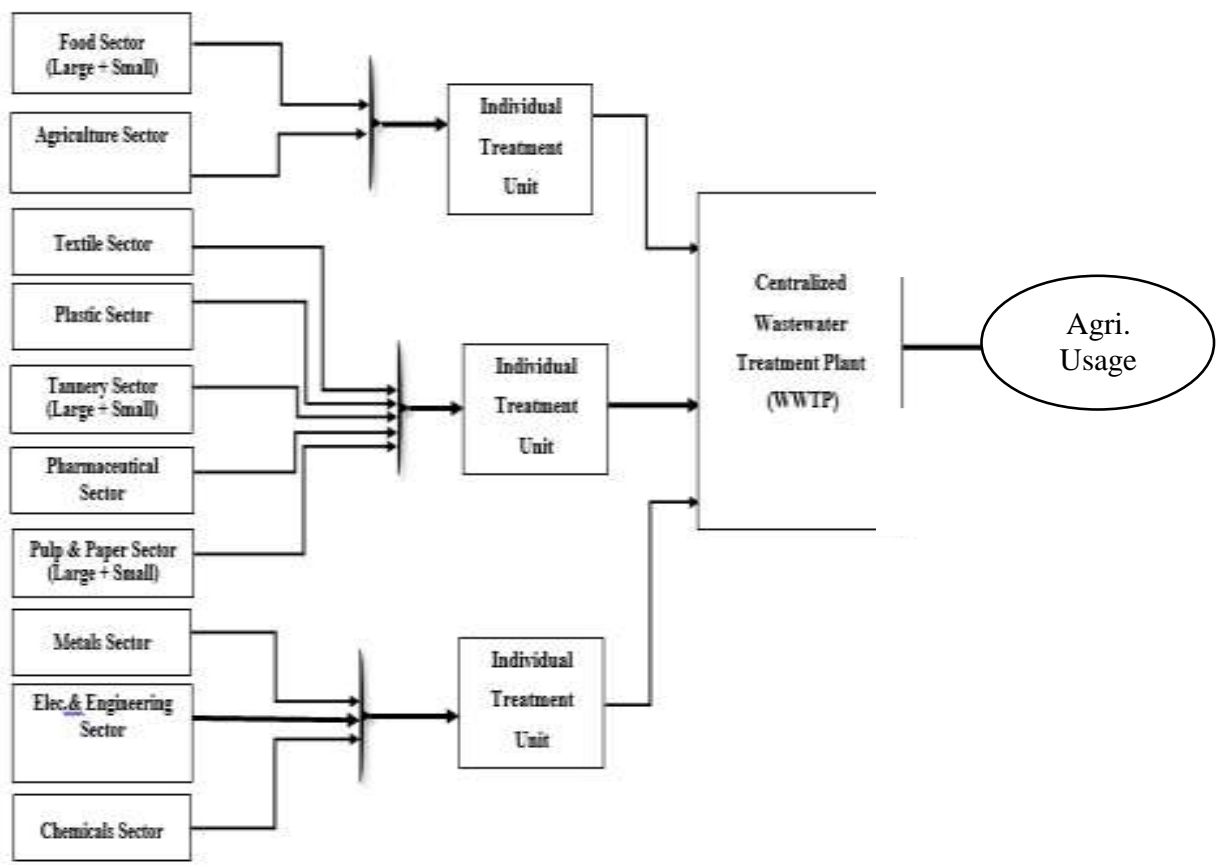

Figure (2): Scheme for Scenario (2) 
J. Environ. Sci.

Institute of Environmental Studies and Research - Ain Shams University

Table (4): The estimated IWW load and max. flow rate entered the 3 individual treatment units for Scenario 2

\begin{tabular}{|c|c|c|c|c|c|c|c|c|c|c|}
\hline $\begin{array}{c}\text { Group } \\
\text { (1) }\end{array}$ & & 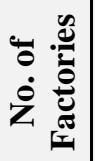 & 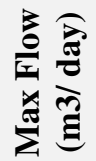 & 包 & 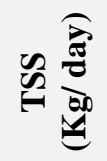 & ڤొ & อิ & 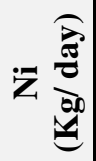 & 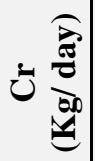 & 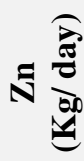 \\
\hline \multirow{3}{*}{$\begin{array}{c}\text { Large } \\
\text { Food } \\
\text { Sector }\end{array}$} & Min & \multirow{3}{*}{3} & \multirow{3}{*}{900} & 504 & 720 & 360 & 675 & 0 & 0 & 0 \\
\hline & Max & & & 1944 & 1845 & 1467 & 3103.20 & 0 & 0 & 0 \\
\hline & Ave & & & 1181.93 & 1287.38 & 909.48 & 1875.52 & 0 & 0 & 0 \\
\hline \multirow{3}{*}{$\begin{array}{c}\text { Small } \\
\text { Food } \\
\text { Sector }\end{array}$} & Min & \multirow{3}{*}{27} & \multirow{3}{*}{945} & 573.48 & 107.33 & 582.39 & 1358.78 & 0 & 0 & 0 \\
\hline & Max & & & 1334.34 & 344.25 & 1168.83 & 3178.04 & 0 & 0 & 0 \\
\hline & Ave & & & 880.57 & 815.54 & 847.17 & 2094.61 & 0 & 0 & 0 \\
\hline \multirow{3}{*}{$\begin{array}{l}\text { Agricult } \\
\text { ure } \\
\text { Sector }\end{array}$} & Min & \multirow{3}{*}{14} & \multirow{3}{*}{2520} & 1734.60 & 1734.60 & 5744.76 & 8506.40 & 0 & 0 & 0 \\
\hline & Max & & & 2472.12 & 2227.68 & 7396.20 & 10962 & 0 & 0 & 0 \\
\hline & Ave & & & 2096.62 & 1988.37 & 6611.82 & 9805.19 & 0 & 0 & 0 \\
\hline \multirow{3}{*}{$\begin{array}{c}\text { Total } \\
\text { (1) }\end{array}$} & Min & \multirow{3}{*}{44} & \multirow{3}{*}{4365} & 2812.08 & 2561.93 & 6687.15 & 10540.18 & 0 & 0 & 0 \\
\hline & Max & & & 5750.46 & 4416.93 & 10032.03 & 17243.24 & 0 & 0 & 0 \\
\hline & Ave & & & 4281.27 & 3489.43 & 8359.59 & 13891.71 & 0 & 0 & 0 \\
\hline
\end{tabular}

Followed Table (4): The estimated IWW load and max. flow rate entered the

3 individual treatment units for Scenario 2

\begin{tabular}{|c|c|c|c|c|c|c|c|c|c|c|}
\hline $\begin{array}{c}\text { Group } \\
\text { (2) }\end{array}$ & & 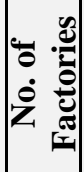 & 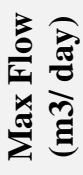 & 胥 & $\underset{\mathscr{E}}{\hat{E}}$ & 尚 & 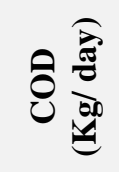 & $\bar{z} \frac{\widehat{\vec{z}}}{\underline{\partial}}$ & $\dot{\vec{\theta}}$ & $\hat{\mathrm{N}}$ \\
\hline \multirow{3}{*}{$\begin{array}{l}\text { Pharm } \\
\text { Sector }\end{array}$} & Min & \multirow{3}{*}{5} & \multirow{3}{*}{1800} & 834.40 & 1168 & 1452 & 4192 & 0 & 0 & 0 \\
\hline & Max & & & 1222.77 & 12274.50 & 7992 & 16143.75 & 0 & 0 & 0 \\
\hline & Ave & & & 1088.53 & 7684.23 & 4992.24 & 10231.81 & 0 & 0 & 0 \\
\hline \multirow{3}{*}{$\begin{array}{l}\text { Plastic } \\
\text { Sector }\end{array}$} & Min & \multirow{3}{*}{19} & \multirow{3}{*}{760} & 3170.91 & 5856.18 & 4950.45 & 8196.60 & 0 & 0 & 0 \\
\hline & Max & & & 3531.72 & 7815.08 & 6606.68 & 10940.20 & 0 & 0 & 0 \\
\hline & Ave & & & 3366.10 & 6677.69 & 5636.94 & 9343.81 & 0 & 0 & 0 \\
\hline \multirow{3}{*}{$\begin{array}{c}\text { Large } \\
\text { Tannery } \\
\text { Sector }\end{array}$} & Min & \multirow{3}{*}{3} & \multirow{3}{*}{5100} & 5625 & 8739 & 4437 & 10638 & 0 & 2245.50 & 0 \\
\hline & Max & & & 6415.80 & 9934.80 & 5049 & 12066.60 & 0 & 2524.50 & 0 \\
\hline & Ave & & & 6209.07 & 9604.01 & 4880.47 & 11698.05 & 0 & 2439.44 & 0 \\
\hline \multirow{3}{*}{$\begin{array}{c}\text { Small } \\
\text { Tannery } \\
\text { Sector }\end{array}$} & Min & \multirow{3}{*}{4} & \multirow{3}{*}{1200} & 1250 & 1814.80 & 982 & 2360 & 0 & 499 & 0 \\
\hline & Max & & & 1509.60 & 2220 & 1182 & 2839.20 & 0 & 595.20 & 0 \\
\hline & Ave & & & 1392 & 2026.05 & 1091.01 & 2622.52 & 0 & 546.66 & 0 \\
\hline \multirow{3}{*}{$\begin{array}{c}\text { Large } \\
\text { Pulp \& } \\
\text { paper } \\
\text { Sector }\end{array}$} & Min & \multirow{3}{*}{3} & \multirow{3}{*}{4500} & 31863.75 & 47989.20 & 21845.70 & 32416.20 & 0 & 0 & 0 \\
\hline & Max & & & 38594.25 & 63702 & 22648.50 & 33592.50 & 0 & 0 & 0 \\
\hline & Ave & & & 36685.20 & 44794.71 & 22402.77 & 33247.83 & 0 & 0 & 0 \\
\hline
\end{tabular}


Followed Table (4): The estimated IWW load and max. flow rate entered the 3 individual treatment units for Scenario 2

\begin{tabular}{|c|c|c|c|c|c|c|c|c|c|c|}
\hline Group (2) & & 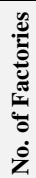 & 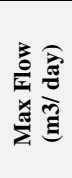 & 菙产 & 象 & 角 & ชิ & 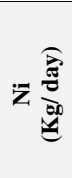 & U & s: \\
\hline \multirow{3}{*}{$\begin{array}{c}\text { Small } \\
\text { Pulp \& } \\
\text { paper } \\
\text { Sector } \\
\end{array}$} & Min & \multirow{3}{*}{10} & \multirow{3}{*}{350} & 2086.26 & 3514.70 & $\begin{array}{c}1608.96 \\
\end{array}$ & 2382.40 & $\overline{0}$ & $\overline{0}$ & $\overline{00}$ \\
\hline & Max & & & 3265.15 & 3977.82 & 1761.55 & 2612.75 & 0 & 0 & 0 \\
\hline & Ave & & & 2625.50 & 3734.14 & 1712.48 & 2541.54 & 0 & 0 & 0 \\
\hline \multirow{3}{*}{$\begin{array}{l}\text { Textile } \\
\text { Sector }\end{array}$} & Min & \multirow{3}{*}{28} & \multirow{3}{*}{840} & 2205 & 6720 & 1170.40 & 2394 & 0 & 0 & 0.21 \\
\hline & Max & & & 2657.76 & 17220 & 1404.48 & 2874.48 & 0.01 & 0.01 & 0.26 \\
\hline & Ave & & & 2473.70 & 11442.64 & 1304.45 & 2634.24 & 0 & 0.01 & 0.23 \\
\hline \multirow{3}{*}{$\begin{array}{l}\text { Total } \\
\text { (2) }\end{array}$} & Min & \multirow{3}{*}{72} & \multirow{3}{*}{14550} & 47035.32 & 75801.88 & 36446.51 & 62579.20 & 0 & 2744.50 & 0.21 \\
\hline & Max & & & 57197.05 & 117144.20 & 46644.21 & 81069.48 & 0.01 & 3119.71 & 0.26 \\
\hline & Ave & & & 52116.19 & 96473.04 & 41545.36 & 71824.34 & 0 & 2932.10 & 0.23 \\
\hline
\end{tabular}

Followed Table(4): The estimated IWW load and max. flow rate entered the 3 individual treatment units for Scenario 2

\begin{tabular}{|c|c|c|c|c|c|c|c|c|c|c|}
\hline Group (3) & & 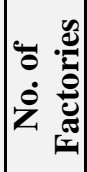 & 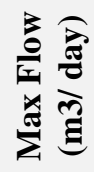 & 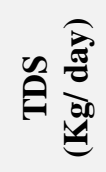 & 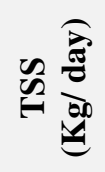 & ஜ & $0 \frac{\vec{\sigma}}{0}$ & 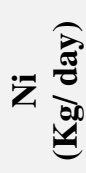 & 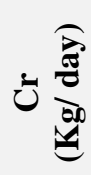 & 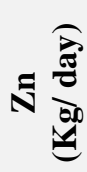 \\
\hline \multirow{3}{*}{$\begin{array}{c}\text { Chemicals } \\
\text { Sector }\end{array}$} & Min & \multirow{3}{*}{11} & \multirow{3}{*}{3,850} & 4090.24 & $4 \quad 4147.61$ & 1669.36 & 2688.84 & 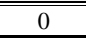 & 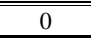 & 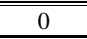 \\
\hline & Max & & & 5120.50 & 5882.80 & 2079 & 3349.50 & 0 & 0 & 0 \\
\hline & Ave & & & 4473.52 & 5043.53 & 1814.83 & 2924.30 & 0 & 0 & 0 \\
\hline \multirow{3}{*}{$\begin{array}{c}\text { Electric \& } \\
\text { Engineering } \\
\text { Sector }\end{array}$} & Min & \multirow{3}{*}{13} & \multirow{3}{*}{650} & 318.73 & 188.37 & 166.73 & 258.51 & 3.73 & 0.01 & 3.33 \\
\hline & Max & & & 624 & 331.50 & 211.25 & 344.50 & 5.07 & 0.04 & 4.27 \\
\hline & Ave & & & 378.80 & 274.27 & 187.59 & 313.63 & 4.52 & 0.02 & 3.95 \\
\hline \multirow{3}{*}{ Metals Sector } & Min & \multirow{3}{*}{10} & \multirow{3}{*}{600} & 3947.40 & 2135.16 & 969.30 & 1453.14 & 0.01 & 262.98 & 0.27 \\
\hline & Max & & & 4398 & 2371.20 & 1081.20 & 1620 & 0.06 & 302.40 & 0.42 \\
\hline & Ave & & & 4165.23 & 2251.88 & 1023.23 & 1533.46 & 0.01 & 280.56 & 0.35 \\
\hline \multirow{3}{*}{$\begin{array}{c}\text { Total } \\
\text { (3) }\end{array}$} & Min & \multirow{3}{*}{34} & \multirow{3}{*}{5,100} & 8356.37 & 6471.14 & 2805.39 & 4400.49 & 3.74 & 262.99 & 3.60 \\
\hline & Max & & & 10142.50 & 8585.50 & 3371.45 & 5314 & 5.13 & 302.44 & 4.69 \\
\hline & Ave & & & 9249.44 & 7528.32 & 3088.42 & $\begin{array}{l}4857.24 \\
\end{array}$ & 4.44 & 282.71 & 4.14 \\
\hline
\end{tabular}


J. Environ. Sci.

Institute of Environmental Studies and Research - Ain Shams University

Table (5): The estimated IWW parameters concentration entering the 3 treatment units and their outputs

\begin{tabular}{||c|c|c|c|c|c|c|c||}
\hline $\begin{array}{c}\text { The First } \\
\text { Treatment Unit for } \\
\text { Group (1) }\end{array}$ & TDS & TSS & BOD5 & COD & Ni & Cr & Zn \\
\hline \hline $\begin{array}{c}\text { Concentration (inlet) } \\
\text { (mg/ ) }\end{array}$ & 1317.40 & 1011.90 & 2298.29 & 3950.34 & 0 & 0 & 0 \\
\hline $\begin{array}{c}\text { Pre-Treatment Unit } \\
\text { (mg/ l) }\end{array}$ & 1317.40 & 708.33 & 1723.72 & 2962.76 & 0 & 0 & 0 \\
\hline $\begin{array}{c}\text { Phyisco-Chemical } \\
\text { Treatment Unit (1) } \\
\text { (mg/ ) }\end{array}$ & 790.44 & 177.08 & 948.04 & 1629.52 & 0 & 0 & 0 \\
\hline $\begin{array}{c}\text { Biological Treatment } \\
\text { Unit (1) (mg/ })\end{array}$ & 434.74 & 8.85 & 47.40 & 81.48 & 0 & 0 & 0 \\
\hline $\begin{array}{c}\text { Concentration } \\
\text { (Outlet) (mg/ 1) of } \\
\text { Drain Law 48/82 for } \\
\text { agriculture }\end{array}$ & 1000 & 30 & 30 & 50 & 0.1 & 0.05 & 2 \\
\hline Removal Eff. (\%) & 67 & 99.13 & 97.94 & 97.94 & - & - & - \\
\hline
\end{tabular}

Followed Table (5): The estimated IWW parameters concentration entering the 3 treatment units and their outputs

\begin{tabular}{|c|c|c|c|c|c|c|c|}
\hline $\begin{array}{l}\text { The First Treatment } \\
\text { Unit for Group (2) }\end{array}$ & TDS & TSS & BOD5 & COD & $\mathbf{N i}$ & $\mathrm{Cr}$ & Zn \\
\hline $\begin{array}{c}\text { Concentration (inlet) } \\
(\mathrm{mg} / \mathrm{l})\end{array}$ & 3931.07 & 8051.15 & 3205.79 & 5571.79 & 0 & 214.41 & 0.02 \\
\hline $\begin{array}{c}\text { Pre-Treatment Unit } \\
(\mathrm{mg} / \mathrm{l})\end{array}$ & 3931.07 & 5635.80 & 2404.34 & 4178.84 & 0 & 214.41 & 0.02 \\
\hline $\begin{array}{c}\text { Phyisco-Chemical } \\
\text { Treatment Unit (1) } \\
(\mathrm{mg} / \mathrm{l})\end{array}$ & 2358.64 & 1408.95 & 1322.39 & 2298.36 & 0 & 10.72 & 0 \\
\hline $\begin{array}{c}\text { Biological Treatment } \\
\text { Unit (1) }(\mathrm{mg} / \mathrm{l})\end{array}$ & 1297.25 & 70.45 & 66.12 & 114.92 & 0 & 0.54 & 0 \\
\hline $\begin{array}{l}\text { Tertiary Treatment } \\
\text { Unit }(\mathrm{mg} / \mathrm{l})\end{array}$ & 778.35 & 17.61 & 36.37 & 63.20 & 0 & 0.03 & 0 \\
\hline $\begin{array}{c}\text { Concentration } \\
\text { (Outlet) }(\mathrm{mg} / \mathrm{l}) \text { of } \\
\text { Drain Law } 48 / 82 \text { for } \\
\text { agriculture }\end{array}$ & 1000 & 30 & 30 & 50 & 0.10 & 0.05 & 2 \\
\hline $\begin{array}{l}\text { Removal Eff. }(\% \eta) \\
\text { Final }\end{array}$ & 80.20 & 99.78 & 98.87 & 98.87 & - & 99.99 & 99.99 \\
\hline
\end{tabular}


Followed Table (5): The estimated IWW parameters concentration entering the 3 treatment units and their outputs

\begin{tabular}{||c|c|c|c|c|c|c|c||}
\hline $\begin{array}{c}\text { The First Treatment } \\
\text { Unit for Group (3) }\end{array}$ & TDS & TSS & BOD5 & COD & Ni & Cr & Zn \\
\hline \hline $\begin{array}{c}\text { Concentration (inlet) } \\
\text { (mg/ l) }\end{array}$ & 1988.73 & 1683.43 & 661.07 & 1041.96 & 1.01 & 59.30 & 0.92 \\
\hline $\begin{array}{c}\text { Pre-Treatment Unit (mg/ } \\
\text { 1) }\end{array}$ & 1988.73 & 1178.40 & 495.80 & 781.47 & 1.01 & 59.30 & 0.92 \\
\hline $\begin{array}{c}\text { Phyisco-Chemical } \\
\text { Treatment Unit (1) (mg/ } \\
\text { 1) }\end{array}$ & 1193.24 & 294.60 & 272.69 & 429.81 & 1.01 & 59.30 & 0.92 \\
\hline $\begin{array}{c}\text { Biological Treatment } \\
\text { Unit (1) (mg/ l) }\end{array}$ & 656.28 & 14.73 & 13.63 & 21.49 & 0.35 & 2.97 & 0.32 \\
\hline $\begin{array}{c}\text { Concentration (Outlet) } \\
\text { (mg/ l) of Drain Law } \\
\text { 48/82 for agriculture }\end{array}$ & 1000 & 30 & 30 & 50 & 0.10 & 0.05 & 2 \\
\hline Removal Eff. (\%ๆ) Final & 67 & 99.13 & 97.94 & 97.94 & 65 & 95 & 65 \\
\hline
\end{tabular}

Table (6): The estimated IWW parameters concentration entering the centralized IWWTP and its outputs

\begin{tabular}{|c|c|c|c|c|c|c|c|}
\hline Centralized IWWTP & Zn & Cr & Ni & COD & BOD5 & TSS & TDS \\
\hline \hline Concentration inlet (mg/ 1) & 0.07 & 0.65 & 0.08 & 57.67 & 33.54 & 15.41 & 689.97 \\
\hline $\begin{array}{c}\text { Biological Treatment Unit (1) } \\
\text { (mg/ l) }\end{array}$ & 0.02 & 0.03 & 0.03 & 2.88 & 1.68 & 0.77 & 379.48 \\
\hline $\begin{array}{c}\text { Concentration Outlet (mg/ l) } \\
\text { of Drain Law 48/82 for } \\
\text { agriculture }\end{array}$ & 2 & 0.05 & 0.10 & 50 & 30 & 30 & 1000 \\
\hline Removal Eff. (\%ๆ) & 65 & 95 & 65 & 95 & 95 & 95 & 45 \\
\hline
\end{tabular}

A final evaluation covers both technical \& financial evaluation. There are different methods to include both evaluations for the total evaluation. The most applied method especially in this branch of work is to calculate the effect of technical evaluation on the financial amount to get the final real cost by dividing the financial budget of each sector on its technical evaluation ratio as illustrated in the following tables. Table (7) illustrated the technical comparison for the applied two scenarios, while table (8) illustrated the 
financial comparison between them to know the best solution economically taking into consideration different financial cost referred to market prices in August 2018 for construction cost, operation cost and maintenance cost.

Finally, table (9) illustrated the total evaluation results where scenario 2 (the industrial sectors having similar industrial wastewater characteristics were grouped together to be treated before being discharged to the centralized IWWTP) has the smallest total cost value after applying the technical evaluation $(600,500,000 \mathrm{EGP})$, followed by scenario 1 (all factories of different industrial sectors discharge their industrial wastewater directly to the centralized IWWTP without any pretreatment) which has the second smallest total cost after applying the technical evaluation which is $(609,787,234 \mathrm{EGP})$.

Table (7): Technical Comparison between Applied Two Scenarios

\begin{tabular}{|c|c|c|c|c|c|c|}
\hline No. & בComparison Face & Wt. & Scenario 1 & Wt. & Scenario 2 & Wt. \\
\hline 1 & Skills needed & 10 & High & 7 & medium & 5 \\
\hline 2 & Energy needed & 15 & High & 4 & medium & 8 \\
\hline 3 & Efficiency & 10 & High & 8 & Very high & 10 \\
\hline 4 & Labors number & 10 & Low & 8 & medium & 6 \\
\hline 5 & Required area (m2) & 10 & medium & 7 & High & 5 \\
\hline 6 & $\begin{array}{c}\text { Operations and } \\
\text { maintenance needed }\end{array}$ & 15 & Very high & 3 & Low & 13 \\
\hline 7 & Control & 5 & Low & 1 & High & 5 \\
\hline 8 & Life time & 10 & Low & 2 & High & 9 \\
\hline 9 & Stability & 5 & Low & 1 & High & 4 \\
\hline 10 & $\begin{array}{l}\text { Mechanical equipment } \\
\text { needed }\end{array}$ & 5 & medium & 3 & High & 4 \\
\hline 11 & $\begin{array}{c}\text { Time for settlement of } \\
\text { IWWTP }\end{array}$ & 5 & medium & 3 & medium & 3 \\
\hline \multicolumn{2}{|c|}{ Total Technical Evaluation } & 100 & & 47 & & 72 \\
\hline \multicolumn{2}{|c|}{ Total Technical Evaluation } & & \multicolumn{2}{|l|}{2} & \multicolumn{2}{|l|}{1} \\
\hline
\end{tabular}


Table (8): Financial Comparison between Applied Two Scenarios

\begin{tabular}{|c|c|c|}
\hline Comparison Item & Scenario 1 & Scenario 2 \\
\hline Initial Cost & $70,000,000$ & $105,000,000$ \\
\hline A- Land & $10,000,000$ & $25,000,000$ \\
\hline B- Construction cost & $30,000,000$ & $40,000,000$ \\
\hline C- Electrical and mechanical cost & $30,000,000$ & $40,000,000$ \\
\hline Operational Cost & $152,100,000$ & $241,360,000$ \\
\hline A- Labors & $46,800,000$ & $56,160,000$ \\
\hline B- Energy & $46,800,000$ & $93,600,000$ \\
\hline C- Spare parts & $39,000,000$ & $60,400,000$ \\
\hline D- Repairing maintenance cost & $19,500,000$ & $31,200,000$ \\
\hline Rehabilitation Cost & $58,500,000$ & $78,000,000$ \\
\hline Loan Cost & $6,000,000$ & $8,000,000$ \\
\hline Total Financial Evaluation/ 20 Years & $286,600,000$ & $432,360,000$ \\
\hline Total Financial Evaluation & 1 & 2 \\
\hline Table (9): Total Evaluation benw Differ & \multicolumn{1}{|l}{} \\
\hline
\end{tabular}

Table (9): Total Evaluation between Different Solutions

\begin{tabular}{|c|c|c|}
\hline Comparison Face & Scenario 1 & Scenario 2 \\
\hline \hline Technical evaluation ratio \% & $47 \%$ & $72 \%$ \\
\hline Total Financial Evaluation/ 20 years & $286,600,000$ & $432,360,000$ \\
\hline Total Cost after applying Technical Evaluation & $609,787,234$ & $600,500,000$ \\
\hline Final Evaluation & 2 & 1 \\
\hline
\end{tabular}

\section{CONCLUSION}

\section{It has been concluded from technical, financial, and total discussion that:}

- In this study (Quesna Industrial Zone) the best solution after several comparisons is Scenario 2 (the industrial sectors having similar industrial wastewater characteristics were grouped together to be treated before being discharged to the centralized IWWTP) for management of IWW, where the total cost after applying the technical evaluation is $(600,500,000 \mathrm{EGP})$ per 20 years. 
- Even Scenario 2 was higher financially than Scenario 1 but after taking the technical comparison into consideration, it became the lowest one in the cost. That shows the high effect of the technical and environmental consideration.

- Prefer in the industrial zones to be from 1 or 2 groups of industry whom connected together with raw materials and the possibility of reuse the byproducts and share some products that minimize the varieties of industrial wastewater and dependency in the cost of its treatment.

- In another industrial zone, another solution could be the best one depending on the industries types, activities, the raw industrial wastewater quality and quantity varieties and the applied treatment solution.

\section{REFERENCES}

American Public Health Authority (APHA) (2009): standard methods for the analysis of water and wastewater.

Ashour, K. (2013): "Treatment of Liquid Wastes Produced by Ceramic Industry', M.Sc. Thesis, Civil Engineering Dep., Ain Shams University, Egypt.

El Hefny, Z. M. (2018): “Upgrading of Existing Dairy Industrial Wastewater Treatment Plant', M.Sc. Thesis, Civil Engineering Dep., Ain Shams University, Egypt.

Khattab, F. M. M. (2012): "Investment Map of Monofeya Governorate", Information Centre, Monofeya Governorate, Ministry of Planning, P 75.

Liu, C. and Côté, R. (2017): A Framework for Integrating Ecosystem Services into China's Circular Economy: The Case of EcoIndustrial Parks. Sustainability, 9(9), 1510. 
Mohamed, S. (2012): 'Treatment of Painting IWW', M.Sc. Thesis, Civil Engineering Dep., Ain Shams University, Egypt.

Saikku, L. (2006): ' 'Eco-Industrial Parks - A Background Report for the EcoIndustrial Park Project at Rantasalmi', Laura Saikku Research Institute for Social Sciences, University of Tampere, Regional Council of Etelä-Savo, Savo, Finland, P 71.

Tessitore, S., Daddi, T. and Iraldo, F. (2015): Eco-Industrial Parks Development and Integrated Management Challenges: Findings from Italy. Sustainability, 7, 10036-10051.

Toll, L. (2009): "Industrial Wastewater Management in Chile - Currently Applied Methods and Suggestions for New Techniques", Department of Information Technology, Uppsala University, P 150 .

Veiga, L. B. E., Alessandra, M. and Szklo, A. S. (2008): Eco Industrial Parks: a tool towards the reduction, reuse and recycling (3R's) of byproducts and wastes: case study in Paracambi EIP, Rio de Janeiro State. Transactions on Ecology and the Environment, 109:395404.

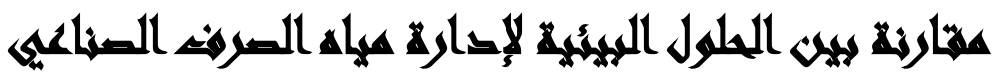

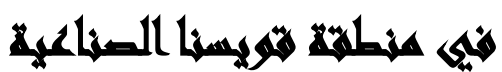

[द]

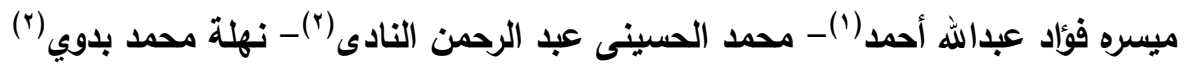

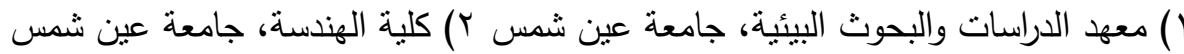

ll

تعد إدارة مياه الصرف الصناعي في مصر أحد الأهداف الرئيسية للتنمية المستدامة. وقد أدت المباء

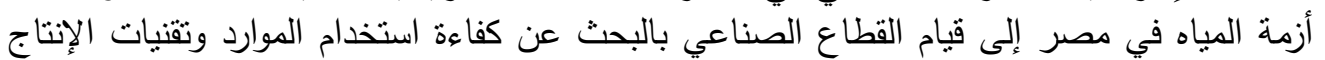

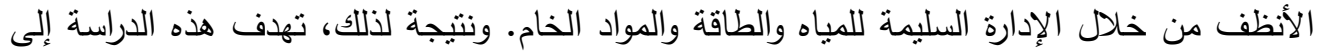

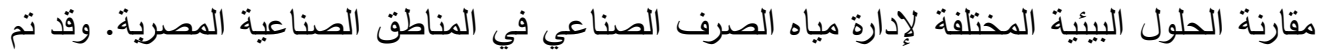

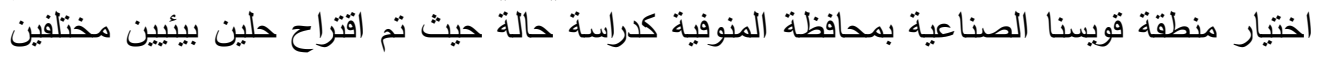
82 
J. Environ. Sci.

Institute of Environmental Studies and Research - Ain Shams University

في هذه الدراسة لإدارة مياه الصرف الصناعي لاختيار الحل البيئي الأفضل لها. ونت أخذ عينات لمباه

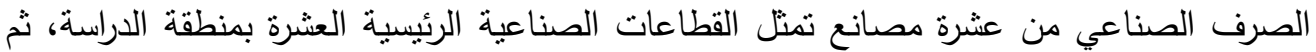

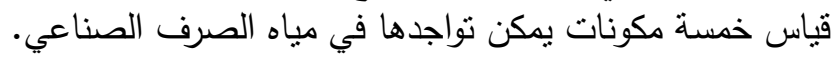

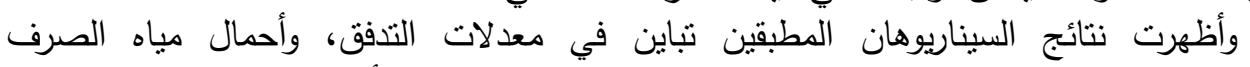

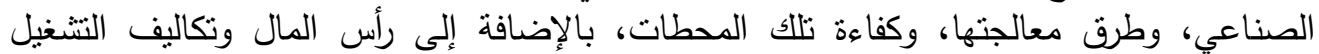

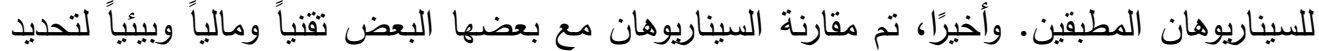

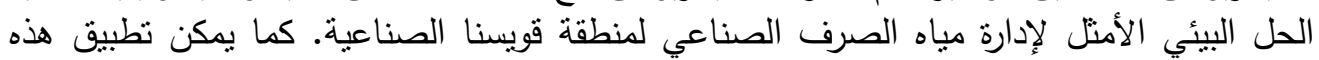
الدراسة على جميع المناطق الصناعية في مصر باستخدام نفس المنهجية المنبعة في هذه الدارة الداسة.

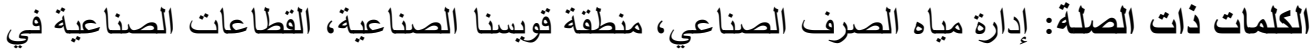

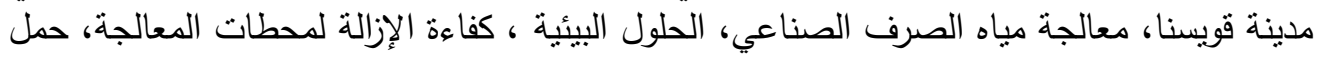
التلوث في مياه الصرف الصناعة مياهي. 